\title{
Indoor Localization Techniques based on Wireless Sensor Networks
}

\author{
Hyo-Sung Ahn ${ }^{1}$ and Wonpil $\mathrm{Yu}^{2}$ \\ ${ }^{1}$ Department of Mechatronics, Gwangju Institute of Science and Technology (GIST) \\ ${ }^{2}$ Electronics and Telecommunications Research Institute (ETRI)
}

Korea

\section{Introduction}

Indoor localization is one of the most important problems in intelligent service robots, and home and office automation. For mobile robot navigation usually vision-based image processing techniques and dead-reckoning techniques based on inertial navigation systems have been used. These traditional technologies however have revealed many problems in actual applications. Vision-based image processing requires landmarks that should be sequentially processed via image detection, feature extraction and scene matching techniques. In actual applications it is hard to extract feature from environment and process data in a real-time on the mobile robot platform. Performance of inertial navigation systems highly depends on the specifications of gyroscope and accelerometer mounted on the robot platform. Measurements from these types of equipment contain various types of random and bias noises. Furthermore since measurement error is usually accumulated, the performance of dead-reckoning system is substantially degraded as time passes. Thus, it is seen that the traditional navigation techniques such as vision and inertial navigation systems are not trustworthy in actual applications.

Recently, with the progress of wireless communication techniques, sensor network-based localization schemes have been actively researched. It is shown that localization techniques on the base of wireless sensor networks are able to overcome many weak points of traditional navigation systems. Wireless sensor network-based localization techniques particularly appear to be beneficial for indoor applications. The main motivation of this chapter is thus to provide a comprehensive overview on state-of-the-art wireless localization networks and a recent progress in this field.

Indoor localization problems are considered much more difficult than outdoor localization problems because GPS signals are not available within buildings or nearby huge structure. Since there are lots of signal interference and signal reflection inside the building, it is even hard to range the signal propagation length using wireless RF signals. Typically in indoor localization, problems of tens-of-meters-long distance or less than ten meters distance are primarily concerns. Thus, if wireless communication signals are contaminated by interference and/or path loss, then the estimated range may include lots of errors. Thus indoor localization is much tougher than outdoor localization. Note that the measurement 
noises and estimated errors on the base of wireless sensor networks (WSN) have different characteristics from vision-based navigation and dead-reckoning systems; that is, measurement errors contained in the measurements of wireless sensor network are not accumulated, which is a case in dead-reckoning systems. Also since wireless sensor networks use wireless communication signals, it is relatively more robust against obstacles than vision-based systems. Moreover, it does not use any landmarks; thus computational load is substantially reduced compared with vision-based systems. However the performance of indoor localization based on wireless sensor network is environmentaldependent. Thus, even though it has many advantages over traditional approaches, it has also weak points.

The main problem considered in this chapter is thus to introduce some novel approaches to handle this problem. The major results presented in this chapter are based on authors' previous works (Ahn \& Yu, 2006; Ahn et al, 20071; Ahn et al, 20072; Ahn et al, 20081; Ahn \& $\mathrm{Yu}, 20082 ; \mathrm{Ahn} \& \mathrm{Yu}, 2008^{3}$; Ahn \& Yu, 20084). Before presenting the alternative approaches developed by the present authors, in the following section we first take a brief review on some relevant works.

\section{Related Works}

There are various aspects in WSN-based indoor localization technologies in terms of communication methodologies, measurement attributes, and communication protocols. In this section we first discuss some recent research trends in this field, and then we categorize indoor localization techniques.

\subsection{Indoor Localization and Navigation}

In the field of WSN-based indoor localization, in fact there is a huge amount of publications. An outstanding survey was carried out in 2001 by (Hightower \& Borriello, 2001) where Active Badge, Active Bat, Cricket, RADAR, MotionStar DC magnetic tracker, etc. were introduced. In (Ssu et al, 2005), they proposed a localization algorithm based on an inspiration from the perpendicular bisector of a chord conjecture. However they developed an algorithm under some restrictive assumptions. In (Peyrard et al, 2000), a localization protocol for mobile stations in a wireless local area network (WLAN; IEEE 802.11) was developed. In their approach the localization was carried out in the scale of extended service set and basic service set (a similar approach can be found in (Elnahrawy et al, 2004)). There also has been lots of research for ultra wideband (UWB; IEEE 802.15.4a)-based localization systems. In particular there have been various research efforts such as outdoor localization application of UWB (Oppermann et al, 2004), UWB localization algorithms (Yu \& Montillet et al, 2006), introduction of a commercialized product (Fontana et al, 2003), channel modelling of UWB signal (Irahhauten et al, 2004), and an overall description about the UWB-based localization (Ingram et al, 2004).

The navigation system is divided into the outdoor navigation and the indoor navigation. For the outdoor case, global positioning system (GPS) can provide reliable positioning information with a favourable accuracy, whereas there is no dominant solution for the indoor object localization. From the literature (Patwari et al, 2005; Sun et al, 2005), it is seen that the indoor navigation can be further categorized into the vision-based navigation system, where the object recognition is a main concern, and the WSN-based navigation 
system, where the signal detection and time synchronization between sensors are important considerations. Recently Federal Communication Commission (FCC) of the USA required the wireless-service providers to accurately locate the position of the 911 caller, and this requirement has accelerated the development of indoor localization systems based on WSN technologies. In this section, as background material, we classify the WSN-based indoor localization systems into several categories.

\subsection{Classification by Measurement Attribute}

Indoor localization technologies have been developed on various concepts and aspects. First, we can consider two different types based on the placement of the main computational unit; client-based systems and server-based systems. In client-based systems the client receives signals from the distributed signal sources. The client is equipped with tags, adapters, or receivers. The client interprets the received-signals as ambient information for localizing its position in a local coordinate system. The server-based methods measure the signals radiated from a client. Then using the signals measured at distributed sensors, the server estimates the position of the client (the fingerprinting can be considered as a server-based approach (Bhargava et al, 2005)). Localization systems based on ad-hoc sensor networks, wherein sensors are distributed in a ubiquitous space as communication nodes, however cannot be categorized as either the client-based or the server-based, because beacons can be placed within a cluster of sensors as reference points. Instead it is a combination of the client-based and the server-based localization systems.

According to the mobility of reference beacons we can also categorize a localization method as one out of the following three: localization systems with fixed-beacons, localization systems with mobile beacons, and beacon-free localization systems. According to the availability of distance information in the localization, we can further differentiate range-based methods from range-free methods, even though most of localization methods can be classified as range-based methods. For a range-free method, see (He et al, 2003). The accuracy of rangefree methods is not good while it provides quite reliable positioning information.

\subsection{Classification by Localization Algorithms}

According to the algorithms used for the localization, we can categorize algorithms into several different types. RSSI (received signal strength index) method uses signal strength arrived, and uses a relationship between the signal-loss and the propagation distance. The following formula is usually used for this relationship:

$$
\mathrm{I}(\mathrm{r})=\mathrm{c} / \mathrm{r}^{\mathrm{a}}
$$

where I(r) represents signal strength at distance $r$ and $c$ can be a constant or environmentdependent variation.

However, the signal attenuation parameter, a, is not fixed, but it depends on the situation. TOA (time of arrival) method uses time-of-flight of the radio signal transmitted. However, in a short distance, it is extremely difficult to calculate the time-of-flight. Furthermore in this method, it is very tough to establish a highly accurate positioning system, since the time synchronization is preliminarily required. To handle the difficulty of this time synchronization, an idea of using the round-trip signal has been proposed (Ping, 2003). However, in this case, usually the size of tag becomes larger because a powerful transceiver is necessary. TDOA (time difference of arrival) method also uses time-of-flight; but it does not 
require the time synchronization between senders and receivers. Instead, it requires time synchronization between receivers. AOA (angle of arrival) method measures the emitteddirection and/or received-direction of signal at fixed reference receivers. Using these directions, the position of the signal source is determined by the triangulation. In TOA and AOA, the array of antennas is an important issue (Sayed et al, 2005), and usually various filtering techniques are used for the signal detection (Gustafsson \& Gunnarsson, 2005). Digital map information-based method (it is called fingerprinting) (Li et al, 2005) makes use of strengths of radio signals radiated from access points (APs). A reference radio map is previously generated based on the signal strengths. When the positions of APs are fixed, the radio map will then not change much, which enables the use of the reference map when estimating the position of a tag. However, note that the radio signal strength is timedependent; hence it is varying even at the same position (Ahn et al, 20072). Thus, the fingerprinting method does not guarantee the accuracy of several meters; instead it can be used for room-level localization (Ahn et al, 20072). Various hybrid methods, which fuse two or more algorithms given above, also have been proposed for a performance improvement (Sayed et al, 2005; Gustafsson \& Gunnarsson, 2005).

However, there are many error sources in the above algorithms. For example, when using TOA and TDOA algorithms, the line-of-sight (LOS) between senders and receivers as well as the timing synchronization between sensors should be ensured, which is usually not satisfied in actual applications. Besides the timing synchronization and LOS, various error sources such as channel fading, shadowing, low signal-to-noise ratios (SNRs), multi-user interference, and multi-path effects deteriorate the performance.

\subsection{Classification by Communication Protocols}

Although various signal/communication techniques such as RFID, laser, sonar, radar, infrared, etc. have been used for the indoor navigation, recently Wi-Fi (IEEE 802.11), UWB (IEEE 802.15.4a), and ZigBee (IEEE 802.15.4) have attracted much attention as introduced in the previous section. Particularly, there have been commercial Wi-Fi and UWB products for the indoor localization. Wi-Fi technology is based on WLAN, which is standardized by IEEE $802.11 \mathrm{a} / \mathrm{b} / \mathrm{g}$ (Rehim, 2004).

In Wi-Fi-based methods, the fingerprinting approach is most popular. However this method needs a reference map that must be generated based on radio signal measurements in advance. Thus, this method is quite sensitive to the environmental variations (Ladd et al, 2004; Xiang et al, 2004). UWB communication is used for WPAN, which is standardized by IEEE 802.15.3a and IEEE 802.15.4a (Gezici et al, 2005; Crepaldi, 2005). UWB system is not much affected by environmental variations, because the signal band width is ultra wide. Thus, recently lots of research and development efforts have been devoted to the UWBbased localization.

In (Guoping \& Rao, 2005), a set of UWB-prototype composed of tag, reader and antenna was developed for the localization purpose. The leading edge of the received-pulse is detected by a maximum likelihood method, which reduces noises and interference effects. However, in this system, the size of UWB transmitter is too big, so it cannot be used for the localization purpose in ubiquitous space. In (Ruiz et al, 2005), a typical structure of UWB chipset was introduced. The typical UWB transmitter is composed of modulator, control digital processor, digital to analog interfaces, pulse generator module, amplifier, filtering, and antenna. The UWB receiver consists of antenna, LNA, a variable attenuator, peak hold 
detector, analog to digital converter, and band pass filter. As explained in (Guoping \& Rao, 2005), UWB-based localization has some advantages over other localization techniques: it uses very narrow pulse ( $\sim 250 \mathrm{ps}$ ) signal; so the signal-arrival instant can be calculated by the receiver accurately if the signal pulse is detected. Also since the narrow pulse signal is immune to multi-paths and free-space fading, UWB is known as an appropriate localization technique in a complicated indoor environment.

However, it is still tough to get rid of multi-paths completely (Bocquet et al, 2005). Moreover, the UWB signal, which ranges from 3.1 to $10.6 \mathrm{GHz}$, coexists with aeronautical and marine radars (Larson et al, 2003); so an appropriate interference suppression method is still necessary when designing transmitter and receiver.

A most challenging problem in the UWB-based localization is the time synchronization between receivers, or between receivers and the transmitter. As an existing technique for this time synchronization, in (Guoping \& Rao, 2005), digital phase lock loop (DPLL) and a lock indicator were used. Actually, it is a toughest thing to ensure the time synchronization of the GHz-UWB systems using the $\mathrm{MHz}$ clock (or $\mathrm{GHz}$ clock) generator. As a solution for this, only the clock alignment between transmitted-signals and receiver clocks was required (Ruiz et al, 2005). In fact, there are numerous publications for the theoretical developments of UWB algorithms as explained in (Lin et al, 2004). However, a successful and practicallyuseful system for localizing any asset has not been reported yet in the literature.

As an alternative to Wi-Fi and UWB, recently IEEE 802.15.4 (ZigBee)-based localization system has been introduced by Chipcon (www.chipcon.com). However, the method used in cc2420 and cc2431 chipsets needs an accurate signal propagation model; hence it is very sensitive to the environmental variation. Probably one of the most advanced technologies in this field is chirp spread spectrum (CSS)-based localization system. A chirp pulse is a frequency modulated signal; it has up-chirp, down-chirp and combination of up-chirp and down-chirp. Since the up-chirp and down-chirp have time-variant frequency, it has a wide bandwidth and uniform power spectral density. Due to the wide bandwidth of CSS, it is highly robust against obstacle, interference, and multi-path. Like UWB, the physical layer of CSS is also standardized by IEEE 802.15.4a.

\section{State-of-the-Art Technologies}

This section presents some technical background and experimental test results of the stateof-the-art WSN-based indoor localizations.

\subsection{Ultra Wide-band (UWB)}

In UWB, there are two different types of signals: Impulse UWB (I-UWB) and multicarrier UWB (MC-UWB). MC-UWB is for the data communication due to its high data rate characteristic. The modulation of $\mathrm{MC}-\mathrm{UWB}$ is based on orthogonal frequency division multiplexing (OFDM). I-UWB does not use a traditional sinusoidal wave for its modulation. Instead, it uses a series of pulse signals whose pulse duration is extremely short, which means it is a ultra wideband in its frequency bandwidth and is suitable for the indoor localization. Generally, however it is still tough to eliminate narrow and wide band noises. Thus, special attentions are required when designing transmitter and receiver (Reed, 2005; Oppermann et al, 2004). In our experimental test, we used Ubisense products (www.ubisense.net), because it was seen that Ubisense has overcome these technical 
challenges in some respects. It is quite straightforward to install Ubisense systems; so a detailed explanation is not given in this chapter. From experimental test (Ahn \& Yu, 2006; Ahn et al, 20071), we found that the Ubisense system can provide about one or two meter accuracy of stationary assets. However, the Ubisense system requires heavy hardware equipment and many cables for data transfer, power, and time synchronization. Also, the hardware size of reference nodes is too big to be used in the indoor service robot.

\subsection{Wi-Fi}

The WLAN technology has an advantage of using license-free radio area as well as of operating in low-power. The WLAN is composed of wireless LAN card, access points (APs), and LAN bridge. The LAN card operates as an interface between network operating system (NOS) and a client like a laptop, and the APs can be seen as LAN hub for interfacing wired network and WLAN area. In WLAN-based localization systems, there are two main issues. The first issue is associated with the size of tag. When monitoring the target, the size of tag (i.e., client) is required to be small such that it can be attached to an object easily. The second issue is related to the generation of a reference radio map. It usually takes a time to generate the radio map and the radio signal strength is a function of time.

For the localization system used in service robot, we have used Positioning Engine 3.1 of Ekahau3 (www.ekahau.com), because it provides a portable tag and user-friendly development software (API), and a nice performance as reported in (Gifford, 2005). Another reason for selecting Ekahau as the sensor network is that it does not require any hardware equipment for its operation. That is, Ekahau is purely software-based localization system. It is also straightforward to use Ekahau system; so a detailed description is not provided in this chapter. From experimental tests (Ahn et al, 20072), we found that WLAN-based localization system can be used for zone-to-zone navigation and room-level localization of stationary objects (Ahn et al, 20072).

\subsection{ZigBee}

The ZigBee communication protocols are defined in IEEE 802.15.4a wireless medium access control (MAC) and physical layer (PHY), and ZigBee Specification generated by ZigBee alliance, Inc. The ZigBee alliance added the network layer (NWT) and the network framework in the application layer based on the IEEE 802.15.4 MAC and PHY. The ZigBee communication is effective for the short range information exchange. The key feature is lowpower and no infrastructure required. Unlike UWB, however ZigBee is not designed for the ranging estimation and positioning. Thus, there are relatively few ZigBee products for indoor localization. Even though the ZigBee signal strength can be used for a range detection purpose, it is difficult to keep the constant signal strength. Also, since the signal strength is dependent on the environmental variation, a fixed signal propagation model cannot be used for a ranging purpose.

\subsection{CSS}

The chirp spread spectrum (CSS)-based communication protocol and chipset technology is shown to dominate the indoor localization market (Ahn et al, 20081). The major advantage of the CSS technology is to provide robust performance for LR-WPAN (low rate wireless personal area network) even in the presence of path loss, multi-path, and reflection. The 
center frequency of chirp pulse is $2.44 \mathrm{GHz}$, chirp duration is $1 \mu \mathrm{s}$, and usaully the chirp bandwidth is $80 \mathrm{MHz}$. Since Doppler effect of chirp signal is ignorable, it is particularly useful for mobile applicaations. Up-chirp or down-chirp signal is detected by autocorrelation of the same signal. When the same signal is auto-correlated with a signal that has been time-synchronized, a pulse will appear. The magnitude of the pulse is dependent on the accuracy of time synchronization between two convoluted signals.

The ranging technique outlined in IEEE 802.15.4a is called symmetric double sided two-way ranging (SDTWR). The SDTWR is based on the time duration that takes for a round-trip between a transmitter and receiver. NanoTron has commercialized a localization chipset that is based on CSS communications. In nanoLOC, the range between a transmitter and receiver is estimated by compensating for reply time within nodes. Recently the real-time one way ranging technique (RT-OWRT) was established, which localizes the tag using oneway communication signals (Ahn et al, 20081).

\subsection{Comments}

In (Ahn et al, 20071; Ahn et al, 20072; Hur \& Ahn, 2008), experimental test results of UWB, $\mathrm{Wi}-\mathrm{Fi}$, ZigBee, CSS are presented. In the case of Ubisense (UWB), overall accuracy is about 1 2 meters when the target is stationary for a long time and Ekahau (Wi-Fi) is only valid for the room-level position estimation. In the case of Chipcon cc2431 (ZigBee), the position is detected within about 5 meters. NanoLOC (CSS) provides localization information with 2 5 meters accuracy. It was shown that the accuracy of localization is highly dependent on the mobility of target. If the target is stationary, the accuracy is improved while if it is mobile, then the accuracy is not reliable.

From experimental tests it is also observed that the performance of localization systems is affected by the environment. Table 1 summarizes the advantage and disadvantage of individual WSN localization system. As shown in this table, no one is a dominant solution for indoor object localization. Also, since the WSN-based localization systems of Table 1 are server-based methods, many tags cannot be tracked at a time.

In the following section, we address a new localization technique that is very cheap to implement and can provide stable position information of stationary objects. Also, since the new method is a client-based localization, many tags can be localized at a time. The new method is developed based on received signal strength index (RSSI) of the radio signal radiating from fixed reference nodes. For the signal strength detection and for obtaining a reliable signal propagation model, the reference tags are used. 


\begin{tabular}{|c|c|c|c|c|}
\hline & Wi-Fi & ZigBee & UWB & CSS \\
\hline Disadvantages & $\begin{array}{l}\text {-Low accuracy } \\
\text {-Slow response } \\
\text {-Signal } \\
\text { interference } \\
\text {-Radio map is } \\
\text { required }\end{array}$ & $\begin{array}{l}\text {-Signal } \\
\text { propagation } \\
\text { model is } \\
\text { required } \\
\text {-Time- } \\
\text { dependent } \\
\text { signal } \\
\text { characteristics }\end{array}$ & $\begin{array}{l}\text {-Heavy } \\
\text { hardware } \\
\text {-Difficult to } \\
\text { locate mobile } \\
\text { objects } \\
\text {-Expensive } \\
\text { equipment }\end{array}$ & $\begin{array}{l}\text {-Expensive } \\
\text { equipment }\end{array}$ \\
\hline Advantages & $\begin{array}{l}\text {-No additional } \\
\text { infrastructure } \\
\text {-Stable position } \\
\text { information }\end{array}$ & $\begin{array}{l}\text {-Simple } \\
\text { hardware } \\
\text {-Small tag size }\end{array}$ & $\begin{array}{l}\text {-Relatively } \\
\text { accurate } \\
\text { localization of } \\
\text { stationary } \\
\text { objects }\end{array}$ & $\begin{array}{l}\text {-Relatively } \\
\text { reliable and } \\
\text { precise } \\
\text { localization }\end{array}$ \\
\hline
\end{tabular}

Table 1: Comparison of performance of WSN-based localization systems

\section{Environmental Adaptive Indoor Localization Techniques}

As mentioned in the previous section, the existing localization technologies have their own disadvantages and advantages. One of the challenging problems in indoor localization based on WSN is the sensitivity of RF signals in indoor environment. To resolve this problem in this section we outline environmental adaptive indoor localization techniques, which were developed in our earlier research works (Ahn \& Yu, 20082; Ahn \& Yu, 20083; Ahn \& Yu, 20084).

This section presents a new indoor localization method based on RSSI in an office environment. The RSSI-based indoor localization has attracted a lot of research recently due to its simplicity and low cost as explained in (Zhang et al, 2006), and due to a less sensitivity to the bandwidth and occurrence of undetected direct path (Hatami \& Pahlavan, 2006). The newly-proposed RSSI-based method consists of reference nodes, which receive and send radio signals, and a tag, which computes its position using radio propagation model updated in real time based on received signal strength. Thus, the newly-proposed method can be considered as a client-based method.

The tag measures received-signal strength and calculates the distance between reference nodes and the tag. The tag is attached to an object that is a target to be tracked, and reference nodes are placed at reference points. A reference node is composed of transmitter and receiver. The receiver computes model parameters based on received-strengths of signals, which are sent from other reference nodes.

The left figure of Figure 1 depicts reference nodes and a tag. This figure shows that the radio signal emitted from a transmitter in a reference node is received by its own receiver, by the receivers in other reference nodes, and by the tag. Even though this figure illustrates three reference nodes, the number of reference nodes and tags generally can be extended to $\mathrm{N}$.

In Equation (1), we need to calculate model parameters a and c based on received signal strengths. If the model parameters a and $c$ are known, then using the measured signal strength I(r), we can reversely calculate the distance between reference nodes and the tag. In what follows, we will explain the algorithm briefly (for a more comprehensive description, refer to $\mathrm{Ahn} \& \mathrm{Yu}, 2008^{4}$ ). The signal attenuation parameter $\mathrm{a}$ is sensitive to the 
environmental variation and the initial signal strength $\mathrm{c}$ is a function of time. It is assumed that the positions of reference nodes are known. Also it is supposed that when the tag and receivers receive signals, they can know where the signal comes from. For convenience, let us say that the receiver of the reference node \#i receives its own signal with strength $c_{i}$ and receives the signal of reference node $\# \mathrm{j}$ with strength $\mathrm{c}_{\mathrm{i}} \mathrm{j}$. Also, the tag receives the signal of the reference node \# $i$ with strength $I_{i}$. The distance between the reference node \# $i$ and the tag is denoted as $r_{i}$, and signal attenuation parameter is $a_{i}$. Then, unknown parameters are $r_{i}$ and $a_{i}$, and measured values are $c_{i}$ and $I_{i}$. Now, the problem is to find $r_{i}$ and $a_{i}$ as accurately as possible using measurements $\mathrm{C}_{\mathrm{i}} \mathrm{j}$ and $\mathrm{I}_{\mathrm{i}}$.
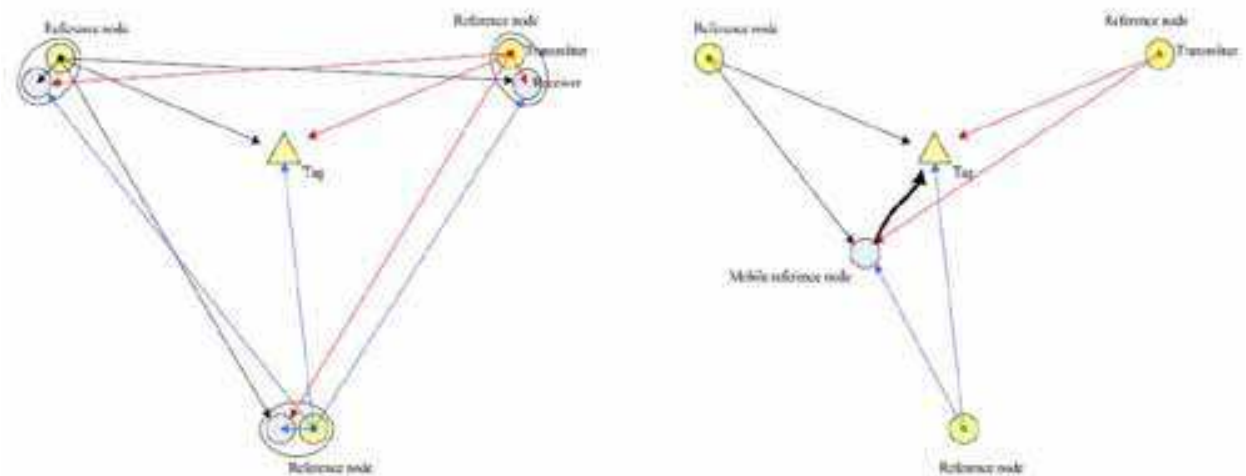

Fig. 1. Left: Localization network composed of three reference nodes (transmitter and receiver; $\mathrm{N}=3$ ) and a tag. Right: Localization network using a mobile reference node.

A simple solution for the parameter estimation is to use the fixed length between the reference node $\# \mathrm{i}$ and the reference node $\# \mathrm{j}, \mathrm{i} \neq \mathrm{j}$. That is, since reference nodes are fixed at known points, and $c_{i}$ and $c_{j}$ are related by the following equation:

$$
\mathrm{c}_{\mathrm{i}} \mathrm{j}=\mathrm{c}_{\mathrm{j}} /\left(\mathrm{r}_{\mathrm{i}}\right)^{\mathrm{a}} \mathrm{a}_{\mathrm{j}}
$$

where $\mathrm{r}_{\mathrm{i}} \mathrm{j}$ is the distance between the reference node $\# \mathrm{i}$ and the reference node $\# \mathrm{j}$, and $\mathrm{c}_{\mathrm{i}} \mathrm{j}$ is a signal strength of the node \# received by the node \#i. Therefore, $a_{j}$ is simply calculated as

$$
a_{j}=\ln c_{j} / c_{i} j / \ln r_{j} j
$$

Thus, attenuation parameters $a_{i}, i=1, \ldots, N$ calculated by Equation (3) are used with together $I_{i}$ for the distance, $r_{i}, i=1, \ldots, N$, calculation in Eq. (1). However, Equation (3) calculates the attenuation parameters in a simple manner and it does not filter out measurement noises. So, as an alternative, we can conceive a method that uses average of received signals. That is, for the calculation of $a_{j}$, we use all $c_{i}, i=1, \ldots, N(i \neq j)$ in the following way:

$$
a_{j}=1 /(N-1) \sum_{i=1, i \neq j}{ }^{N}\left[\ln _{\mathfrak{j}_{j}} / c_{i} j / \ln ^{r_{j} j}\right]
$$

which will remove some high frequency noise components and uncompensated bias errors. The overall procedure can be summarized in the sequel.

The receivers, which are placed in reference nodes, and the tag receives signals radiating from the transmitter of individual reference node. Then, reference nodes send information of measured signal strengths to the tag using wireless local area network so that the tag can estimate $a_{j}$ using Equation (3) or Equation (4). Then, using Equation (1), the tag calculates distances between reference nodes and it such as

$$
r_{i}=\left[c_{i} / I_{i}\right]^{1 / a_{i}}
$$


Now, using Equations (5) it is straightforward to estimate the position of the tag. A nice review on the localization algorithms is presented in (Sayed et al, 2005). In (Sayed et al, 2005), if the positions of reference nodes are known as $\left(\mathrm{x}_{\mathrm{i}}, \mathrm{y}_{\mathrm{i}}\right)$, then the position of the tag is calculated as:

$$
[x, y]^{\mathrm{T}}=\left[\mathrm{H}^{\mathrm{T}} \mathrm{H}\right]^{-1} \mathrm{H}^{\mathrm{T}} \mathrm{b}
$$

where:

$$
\mathrm{H}=\left[\begin{array}{cc}
\mathrm{x}_{2} & \mathrm{y}_{2} \\
\mathrm{x}_{3} & \mathrm{y}_{3} \\
\mathrm{x}_{4} & \mathrm{y}_{4} \\
\vdots & \vdots \\
\mathrm{x}_{\mathrm{N}} & \mathrm{y}_{\mathrm{N}}
\end{array}\right] ; \mathrm{b}=\left[\begin{array}{c}
\mathrm{K}_{2}^{2}-\mathrm{r}_{2}^{2}+\mathrm{r}_{1}^{2} \\
\mathrm{~K}_{3}^{2}-\mathrm{r}_{3}^{2}+\mathrm{r}_{1}^{2} \\
\mathrm{~K}_{4}^{2}-\mathrm{r}_{4}^{2}+\mathrm{r}_{1}^{2} \\
\vdots \\
\mathrm{K}_{\mathrm{N}}^{2}-\mathrm{r}_{\mathrm{N}}^{2}+\mathrm{r}_{1}^{2}
\end{array}\right]
$$

and $\mathrm{K}_{\mathrm{i}}^{2}=\mathrm{xi}^{2}+\mathrm{y}_{\mathrm{i}}^{2}$.

So far, we have presented a localization method using reference nodes which include both transmitter and receiver. The receiver receives radio signal sent from transmitter and it measures the signal strength for calculating signal propagation parameters. However, the communication load may be heavy in this method.

Another simple localization method also can be developed using a reference tag without receivers in reference nodes. The right figure of Figure 1. depicts the wireless localization network that is composed of a mobile reference node, fixed reference nodes, and a tag. The mobile reference node is movable and its position is assumed known. This assumption is valid in a mobile robot application. The mobile robot position is measured from localization sensor network such as StarLITE (Yu, W. et al, 2006; Chae et al, 2005). In the right figure of Figure 1., the tag can be attached to pedestrian.

In this method, the mobile reference node is used for modeling the signal propagation characteristics. The signal propagation is governed by the following formula:

$$
\mathrm{I}_{\mathrm{i}}(\mathrm{r})=\mathrm{A} / \mathrm{r}_{\mathrm{i}}^{\mathrm{a}} \mathrm{I}_{\mathrm{i}}(0)
$$

where $I_{i}(r)$ is the received strength of the $i$-th reference node signal; $r_{i}$ is the distance between the mobile reference node and the $i$-th reference node; and $I_{i}(0)$ is the initial signal strength of the i-th reference node.

Now, the problem is to find the signal attenuation parameter a and proportional constant $A$ based on $\mathrm{I}_{i}(\mathrm{r}), \mathrm{r}_{\mathrm{i}}$, and $\mathrm{I}_{\mathrm{i}}(0)$. Since in this approach the initial signal strengths $\mathrm{I}_{\mathrm{i}}(0)$ are not calculated, $\mathrm{I}_{i}(0)$ should be measured first. The relationship given by Equations (7) can be changed as:

$$
a \ln \mathrm{r}_{\mathrm{i}}=\ln \left[\mathrm{I}_{\mathrm{i}}(0) / \mathrm{I}_{\mathrm{i}}(\mathrm{r})\right]+\ln \mathrm{A}
$$

From Equations (8), we can easily obtain the following relationships:

$$
\begin{gathered}
\mathrm{a}\left(\ln ^{\mathrm{r}_{1}}-\ln ^{\mathrm{r}_{2}}\right)=\ln \left[\mathrm{I}_{1}(0) / \mathrm{I}_{1}(\mathrm{r})\right]-\ln \left[\mathrm{I}_{2}(0) / \mathrm{I}_{2}(\mathrm{r})\right] \\
: \\
\mathrm{a}\left(\ln ^{\mathrm{r}}{ }_{\mathrm{N}-1}-\ln ^{\mathrm{r}} \mathrm{N}\right)=\ln \left[\mathrm{I}_{\mathrm{N}-1}(0) / \mathrm{I}_{\mathrm{N}-1}(\mathrm{r})\right]-\ln \left[\mathrm{I}_{\mathrm{N}}(0) / \mathrm{I}_{\mathrm{N}}(\mathrm{r})\right]
\end{gathered}
$$


Equations (9-10) can be now used for finding the attenuation parameter a. A more accurate estimation can be performed by combination of all measurements. The combination is achieved by the following formula:

Therefore, the parameter a is estimated as:

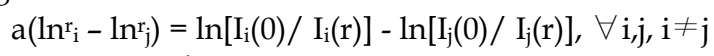

$$
\mathrm{a}=1 / \mathrm{N}(\mathrm{N}-1) \sum_{\mathrm{j}=1} \mathrm{~N} \sum_{\mathrm{i}=1, \mathrm{i}} \mathrm{j}^{\mathrm{N}}\left(\ln \left[\mathrm{I}_{\mathrm{i}}(0) / \mathrm{I}_{\mathrm{i}}(\mathrm{r})\right]-\ln \left[\mathrm{I}_{\mathrm{j}}(0) / \mathrm{I}_{\mathrm{j}}(\mathrm{r})\right]\right) /\left(\ln _{\mathrm{i}}^{\mathrm{r}}-\ln _{\mathrm{j}} \mathrm{r}\right)
$$

The proportional constant $\mathrm{A}$ is obtained by inserting a into Equations (8).

\section{Hardware Development}

For the verification of the localization algorithms described in the previous section, we developed a ubiquitous ZigBee (2.4 GHz RF communication system) sensor network. The reason we selected the ZigBee communication system as our hardware platform is two-fold: First, it is very convenient and cheap to use ZigBee transceiver as reference node. Second, we can easily add a motion sensor to ZigBee system so that the tag senses the motion of object, which can be nicely used for saving the power and for reliable object localization. The ZigBee system developed thus operates in sleep mode and operating mode according to events; hence, the battery life of the tag is relatively longer than other wireless communication systems. We developed fixed reference transmitters and fixed reference tags, which can be used as receivers in the reference nodes. That is, we place the fixed reference tags nearby the reference transmitters. In what follows, we explain the hardware equipment in detail.

\subsection{Fixed Reference Transmitter}

The basic requirements for the fixed reference node are as follows:

- Nodes communicate each other wirelessly and only can be wire-connected to the power outlet

- The user can assign coordination values to individual reference node

- When a battery is used as a power source, it should operates in either sleep mode or active mode based on event

- When there is a request from the moving tag, it must send its coordination to the tag

- The fixed reference node can be easily attached to a wall and a ceiling

- The hardware size is limited to $15 \mathrm{~cm} \times 10 \mathrm{~cm} \times 2 \mathrm{~cm}$

- Each node has its own identity and can encode its identity in the data packet

To satisfy the above requirements, we chose the following components:

- ATmega128L(MCU): 128K Flash, 4K SRAM, 4K EEPROM, 2.56V 3.3V operation volts

- cc2420(RF Transceiver): 2.4 GHz RF communication, 16 Channels, RSSI detection

- SMD Poll type antenna

The firmware and protocol stack are embedded into ATmega128L to manage overall activities of the system. The cc2420 sends and receives data packet based on ATmega128L's control signals. 


\subsection{Mobile Reference Tag}

This mobile reference tag can used as the receiver of individual reference node and can be used for mobile reference node. Thus, it is used for the tag, which can be attached to object. The requirements for the mobile reference tag are:

- To enable the mobile capability, it should be easily mounted on a mobile vehicle like mobile robot, object, pedestrian, etc.

- When it is attached to the mobile robot, it must receive the position of mobile robot through electrical interface (it is assumed that the position of mobile robot is known)

- When there is a request from the other reference tag, it should deliver its position and received-strengths of the signals emitted from the reference nodes

- Individual mobile reference tag has its own identity

- When it is mounted on the mobile robot, the power cable can be connected to the robot

The particular requirements for mobile tag, when it is attached to object, are given as:

- The tag is composed of a motion sensing module and a ZigBee RF module

- The tag operates based on power of the battery

- Physical size is limited as $8 \mathrm{~cm} \times 5 \mathrm{~cm} \times 1 \mathrm{~cm}$ and weight is less than $150 \mathrm{~g}$

- The tag operates in either sleep mode or active mode according to the motion signal detected by the motion sensor

- The tag should receive signals from the fixed reference nodes and from other mobile tag

- The tag is able to calculate the signal propagation parameters in real time

- The calculated signal propagation information should be transmitted to the server, if necessary

The detailed hardware specification developed at ETRI is summarized as follows:

- cc2431(MCU \& RF Transceiver): 128K Flash, 8K SRAM, 4K EEPROM, 250Kbps transmit rate, Multi-purpose general I/O port

- Fractus Chip antenna

- I/O: 2 LEDs, 1 Push button, 1 Reset button, Download Port

Firmware and IEEE 802.15 .4 protocol are embedded on cc2431. Figure 2 is the mobile reference tag developed. In this picture, accelerometer is the motion sensor.

\section{Experimental Results}

For the experimental verification of the algorithm proposed in the previous section (the algorithm described by Equations (2)-(6); let us call this method as the first method), we constructed a wireless sensor network as shown in Figure 3, Figure 4 and Figure 5. From Figure 5, we know that fixed reference nodes, which are composed of fixed reference transmitter and mobile reference tag, are placed at points $(10.5,14.5),(10.5,8.0),(10.5,0.0)$, $(0.0,14.5),(0.0,8.0)$ where the unit of the coordinate value is meter. 


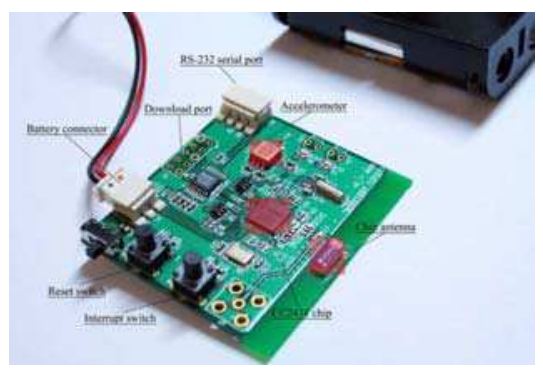

Fig. 2. Mobile reference tag produced.

The fixed-reference transmitter developed does not operate as a transceiver. So, we place mobile reference tag, which can receive signals from the fixed reference transmitter, beside the fixed-reference transmitter so that they (the fixed-reference transmitter and mobile reference tag) work as a reference node in a pair. Note that these nodes are placed at threemeter height above the surface and under the ceiling; but we do not take account of the height.

Figure 3. shows that the reference nodes are composed of mobile reference tag and fixedreference transmitter. The left figure is a reference node attached on the wall and the right figure is a reference node attached under the ceiling. Figure 4 shows the reference nodes installed in office. We place tags at $(6.6,9.8)$ and $(10.5,8.0)$ respectively, as shown in Figure 5. (the height is about 1.6 meters). The place of $(6.6,9.8)$ is within the office and the place of $(10.5,8.0)$ is near the wall.

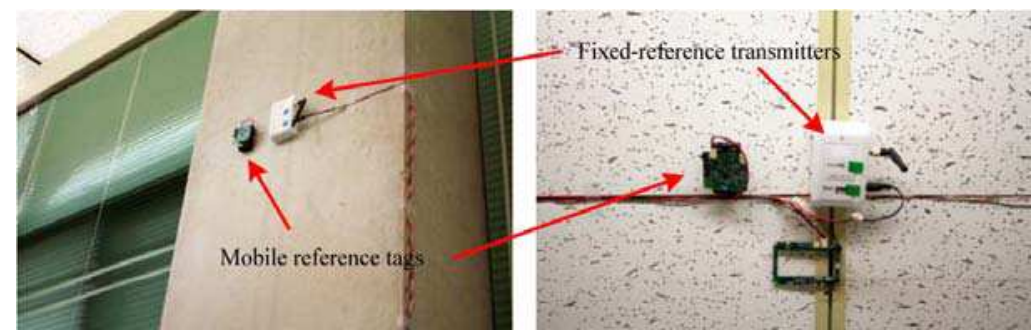

Fig. 3. Fixed reference node is composed of a reference transmitter and a mobile reference tag: the left-top is the node installed in the wall; the right-top is the node installed under the ceiling.

The experiment was performed for 10 minutes. At each sampling time, we obtained a data packet from the tag as shown in Figure 6. Individual mobile reference tag measures RSSI of the signal sent from the fixed-reference transmitters. Then, each mobile reference tag sends the data packet as shown in Figure 6. to the tag. In the packet, the last two data $x$ and $y$ are position of the tag calculated by Chipcon cc 2431 chip mounted in the tag. So, we can compare the estimated-positions of the tag calculated by the proposed-algorithm and by the Chipcon cc2431. 


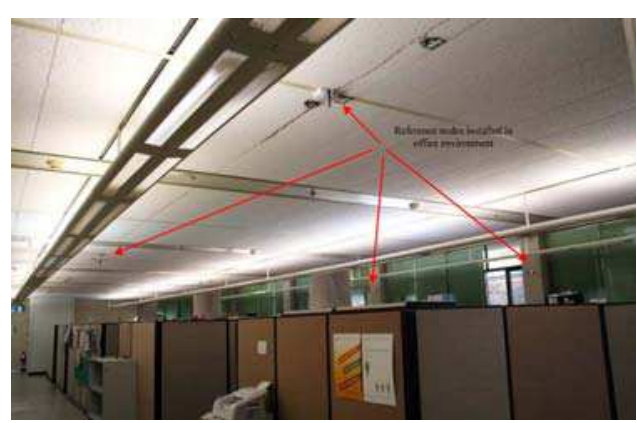

Fig. 4. Fixed reference nodes are installed in office.

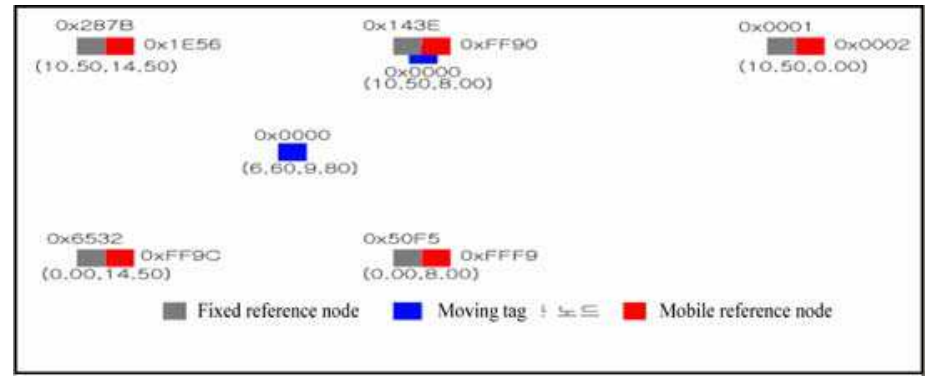

Fig. 5. Fixed reference nodes, moving reference nodes, and tag.

\begin{tabular}{|c|c|c|c|c|c|c|c|}
\hline $\begin{array}{l}\text { Mobile } \\
\text { referenese } \\
\text { node ID }\end{array}$ & $\begin{array}{l}\text { RSSI of } \\
\text { OxpDOAI }\end{array}$ & $\begin{array}{l}\text { RSS1 of } \\
\text { OxI I } 3 \text { B }\end{array}$ & $\begin{array}{l}\text { RSSI of } \\
0 \times 287 \mathrm{~B}\end{array}$ & $\begin{array}{l}\text { RSSI of } \\
0 \times 6532\end{array}$ & $\begin{array}{l}\text { RSS1 of } \\
\text { Oxsops }\end{array}$ & $\mathrm{x}$ & $Y$ \\
\hline
\end{tabular}

Fig. 6. Data packet.

Figure 7-(a) to Figure 7-(f) show experimental test results when the tag is placed at $(6.6,9.8)$. Figure 7-(a) shows the estimated $a_{j}$ from Equation (4). From the plots, it is shown that the attenuation parameters are estimated as about 3 except $a_{4}$, which is estimated as 4 approximately. Figure 7-(b) shows the estimated distances calculated by Equation (5). The dots in Figure 7-(c) are the estimated positions of the tag from the proposed-method and the $\mathbf{x}$-mark represents the actual position of the tag. The dots in Figure 7-(d) show the estimated positions of the tag from cc2431. From Figure 7-(d), we can see that cc2431 estimates the tag position in several fixed points, whereas the proposed-method estimates the position in a random manner.

Since this chapter estimates the stationary object, we recalculate the position based on Figure 7-(c) and Figure 7-(d) in the following way:

$$
x_{n}=1 / n \sum_{i=1}^{n} x_{i}, y_{n}=1 / n \sum_{i=1}^{n} y_{i}
$$

where $x_{i}$ are $y_{i}$ values from Figure 7-(c) and Figure 7-(d). Figure 7-(e) shows the re-calculated $x_{n}$ and $y_{n}$. In Figure 7-(e), the solid lines are the re-calculated $x$ and $y$ of the tag from cc2431; the dot-dashed are the re-calculated $x$ and $y$ of the tag from the proposed method; and the dashed-lines are actual values. From these re-calculated plots, we can see that the proposed method is better in the estimation of $y$; but the cc 2431 is better in the calculation of $\mathrm{x}$. 
To compare the performance quantitatively, we calculate the total errors of Figure 7-(e), which is shown in Figure 7-(f). The total errors are calculated as

$$
e_{n}=\sqrt{\left(x_{n}-x\right)^{2}+\left(y_{n}-y\right)^{2}}
$$

where $x, y$ are true position values of the tag. From Figure $7-(\mathrm{f})$, we can see that the proposed-method has a better performance than cc2431. Figure 8-(a) to Figure 8-(f) show the test result when the tag is placed nearby the wall.

As shown in Figure 8-(f), the proposed-method has a better performance than cc2431. For the experimental test of the algorithm described by Equations (7-12) (let us call this method as the second method), we carried out experimental tests by repetition. For this test, we simply turned-off all mobile reference tags shown in Figures 3-4, and placed a reference mobile reference tag inside the office. This reference tag placed inside the office acts as a mobile reference.
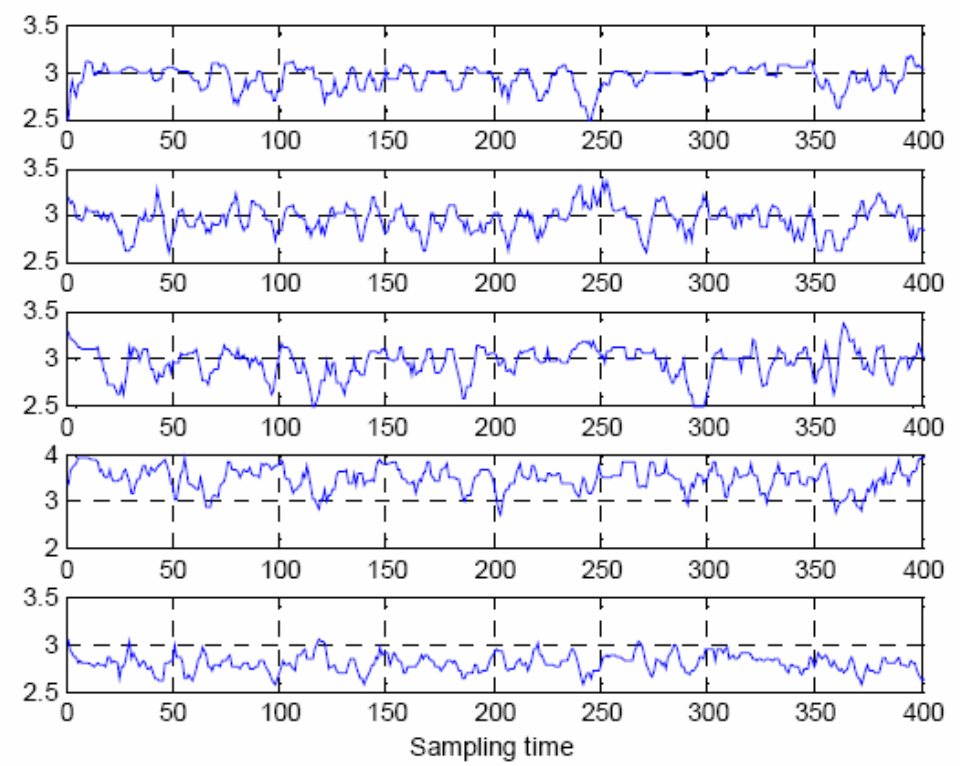

Fig. 7-(a). Experimental test results inside the sensor network. 

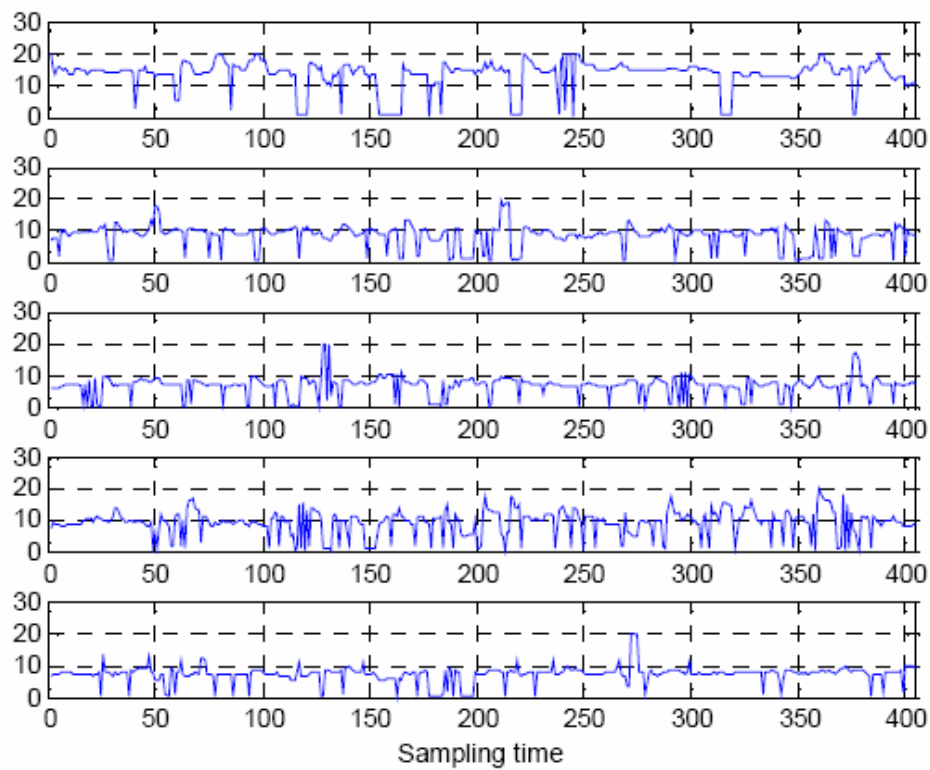

Fig. 7-(b). Experimental test results inside the sensor network.

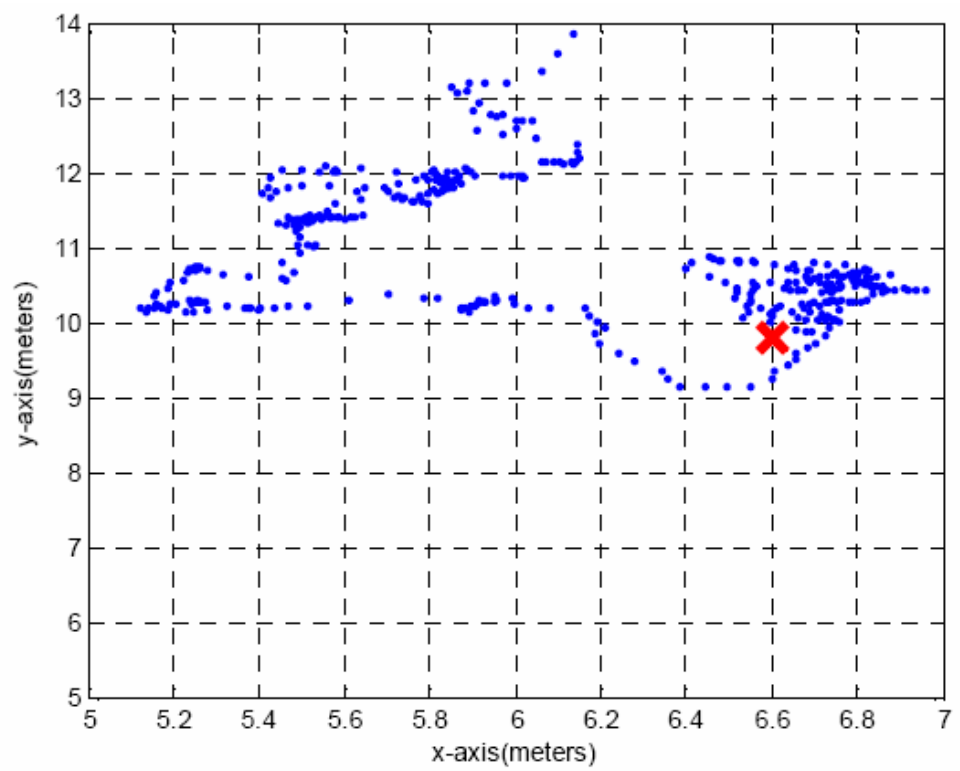

Fig. 7-(c). Experimental test results inside the sensor network. 

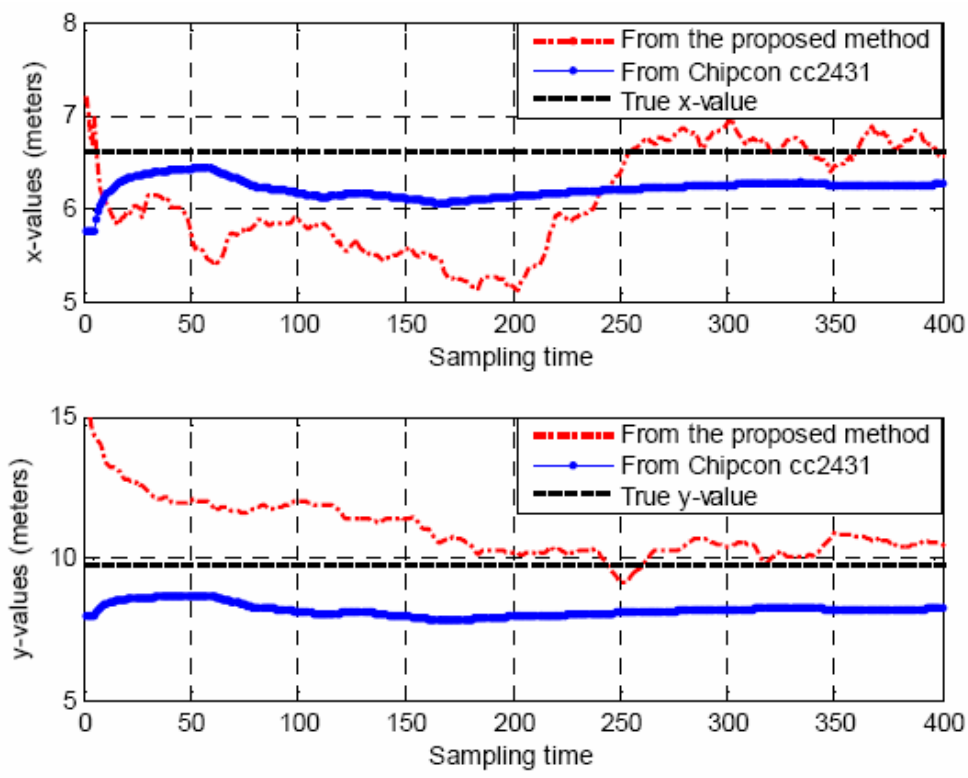

Fig. 7-(d). Experimental test results inside the sensor network.

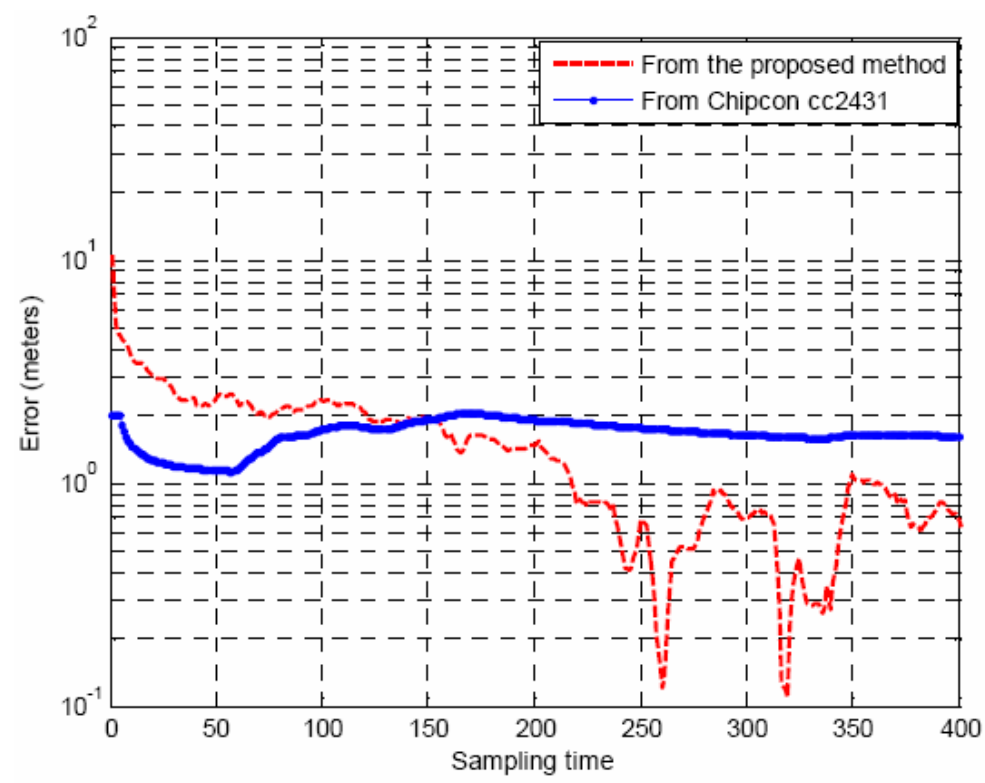

Fig. 7-(e). Experimental test results inside the sensor network. 


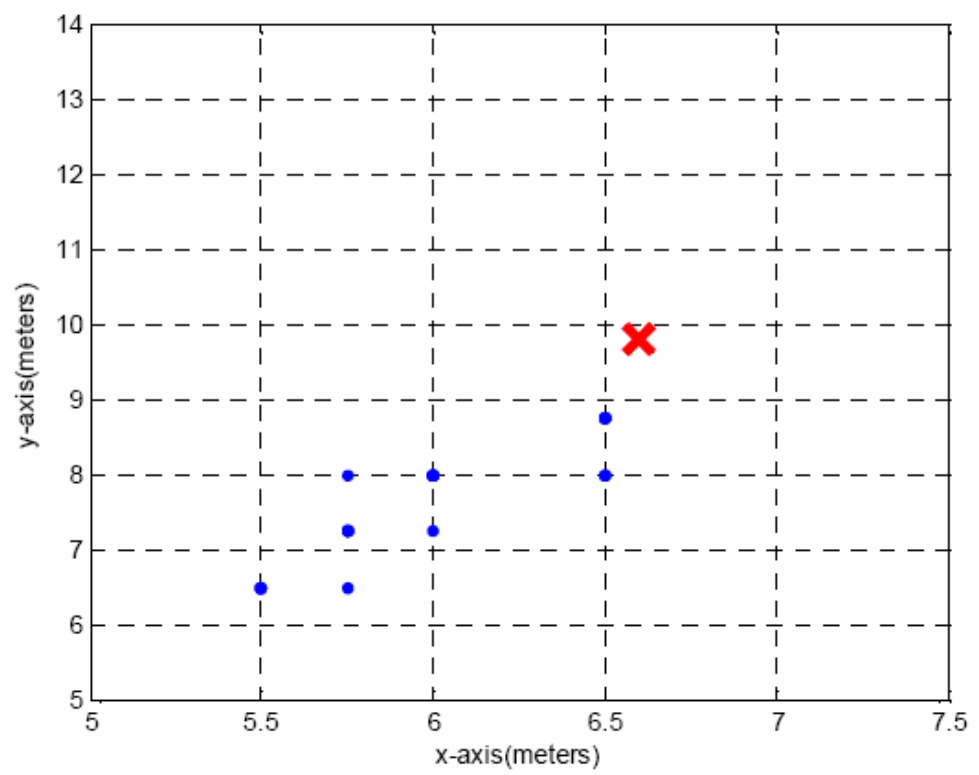

Fig. 7-(f). Experimental test results inside the sensor network.
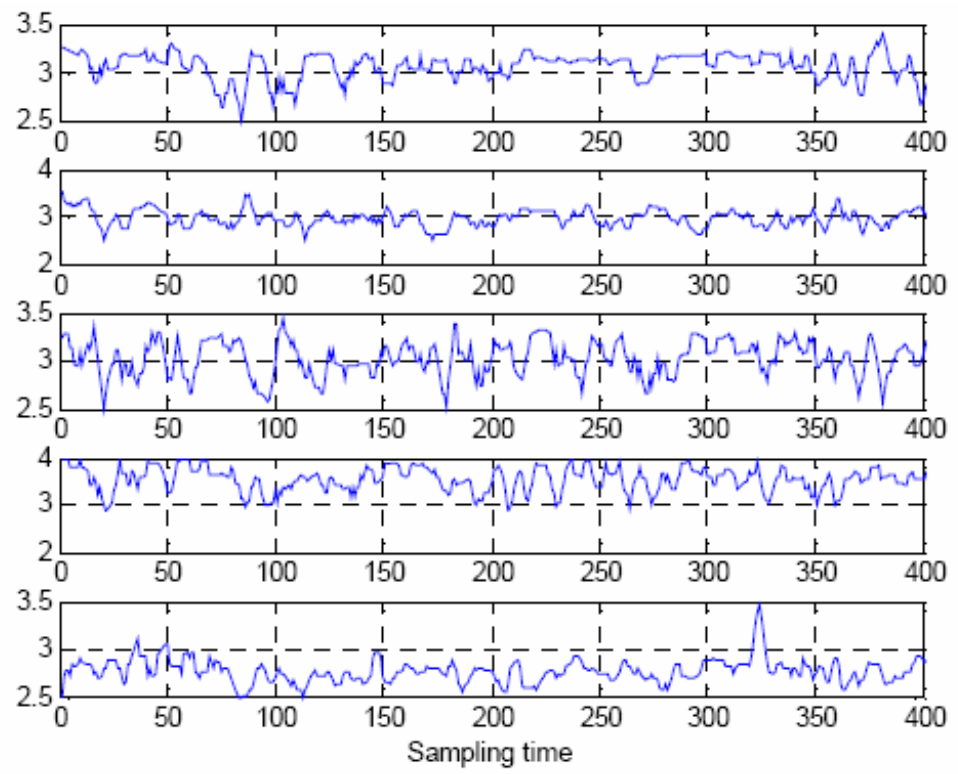

Fig. 8-(a). Experimental test results near the wall. 


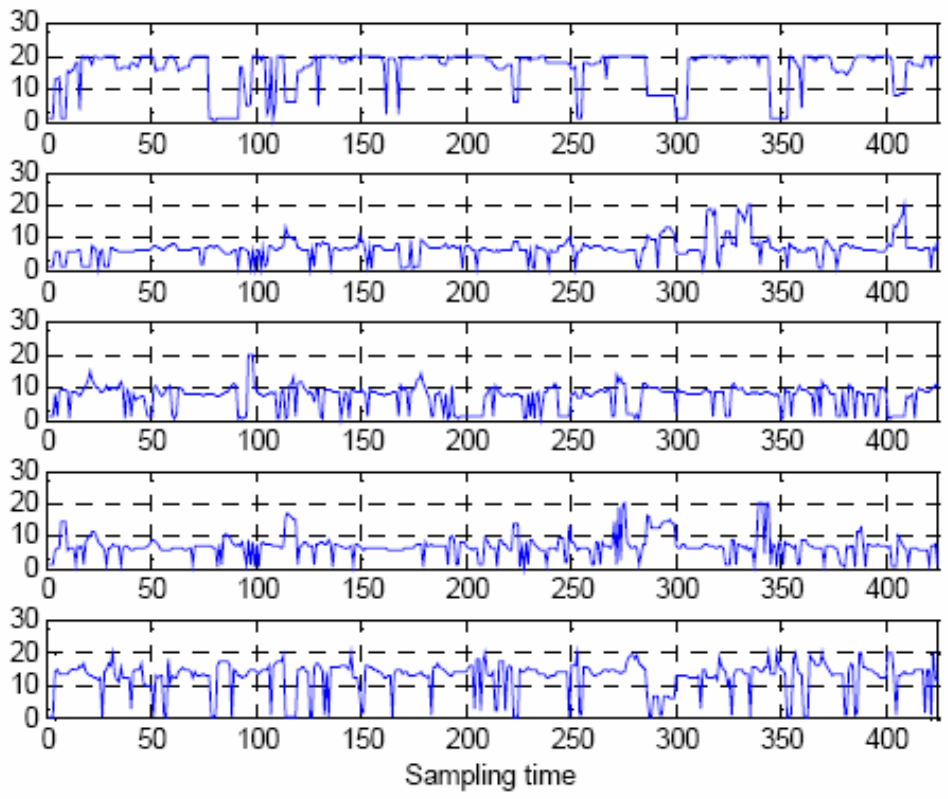

Fig. 8-(b). Experimental test results near the wall.

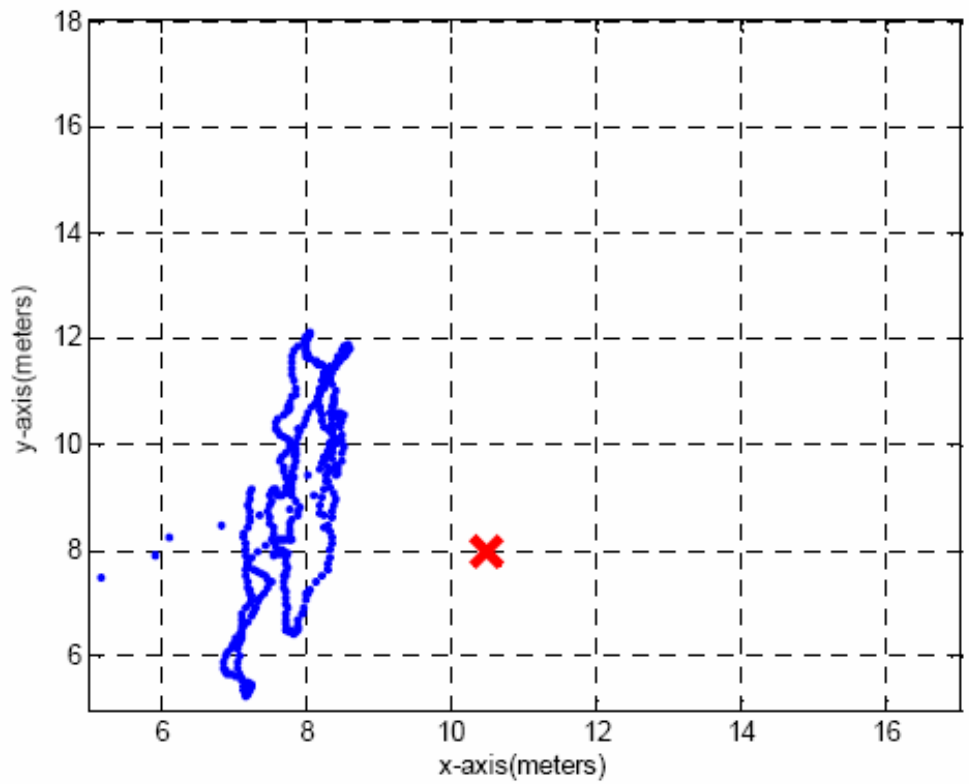

Fig. 8-(c). Experimental test results near the wall. 


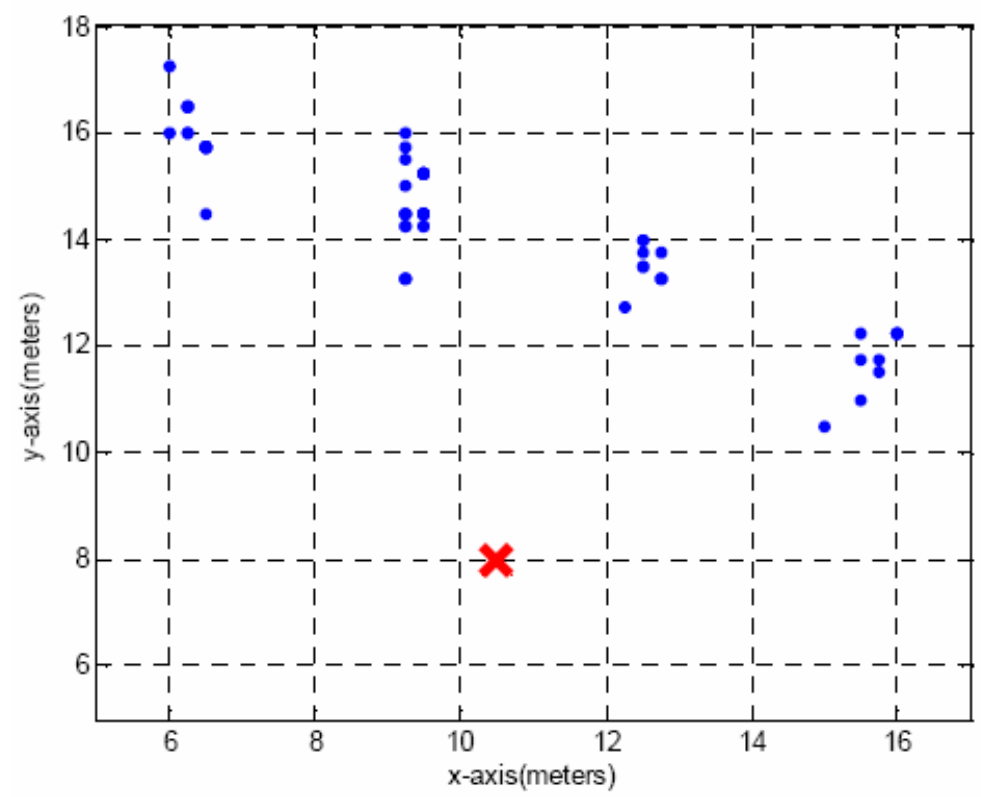

Fig. 8-(d). Experimental test results near the wall.
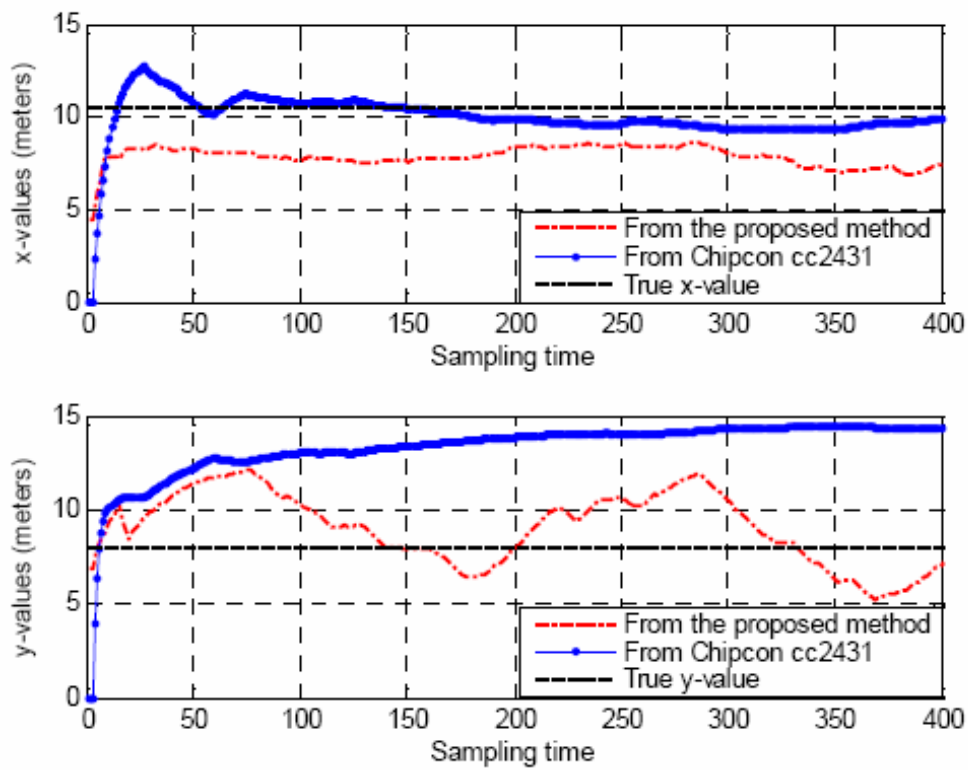

Fig. 8-(e). Experimental test results near the wall. 


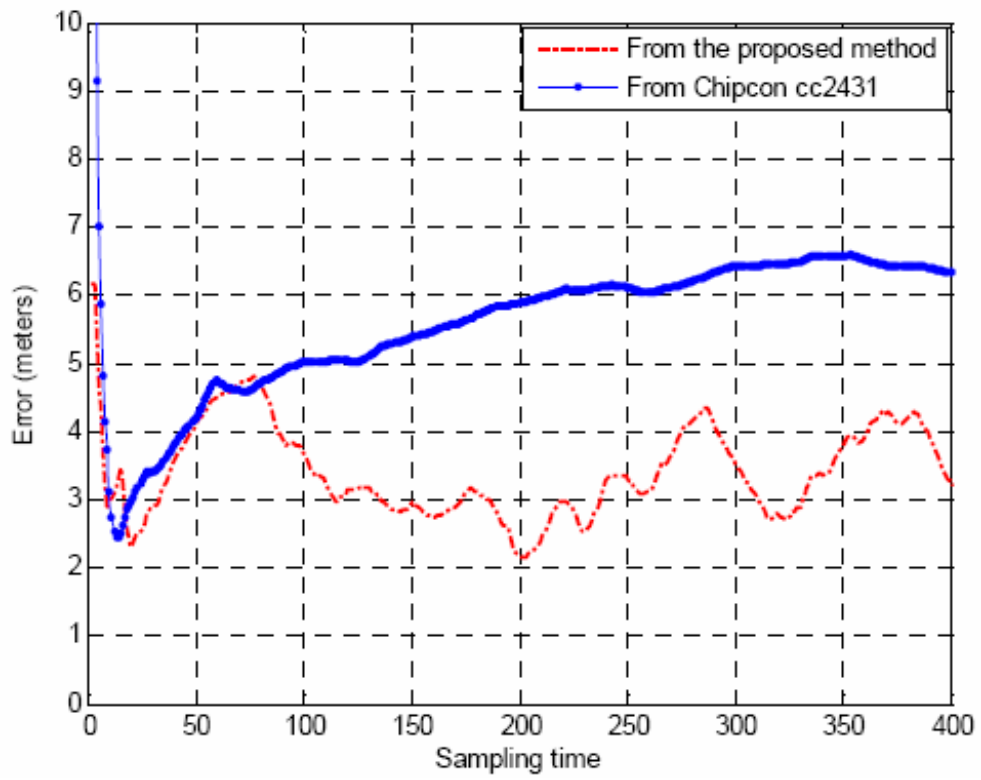

Fig. 8-(f). Experimental test results near the wall.

Table 2 and Table 3 show the test results. The true position is the actual place where the tag was placed. In these tables, MSE stands for mean square error given in Equation (14). From these tables, we observe that the new method has much better performance than cc 2431 in Tests 1, 2, 3, and 4; however cc2431 is slightly better than the new method in Test 5 . Thus, we can see that the new method is dominantly better (four times better out of five tests; 80 percents better) than the commercial cc2431. However, we also note that the second method estimates the position of the tag much faster than the first method. The measured and estimated position values given in Table 2 and Table 3 were sampled at every second, which can be considered as real time estimation.

\section{Conclusions}

In this chapter, we presented a set of classifications of indoor localization techniques. We generated categories according to measurement attribute, location algorithms, and communication protocols. The classifications presented in this chapter provide a compact form of overview on WSN-based indoor localizations. Then, based on the classifications, we introduced server-based and range-based localization systems that can be used for the indoor service robot. Specifically, we presented UWB, Wi-Fi, ZigBee, and CSS-based localization systems.

From actual experimental tests, however we found that the existing WSN-based methods have their own disadvantage. That is, Ubisense system is expensive and needs heavy hardware equipment. The Wi-Fi system (Ekahau) has a low accuracy and is only useful for the room-level localization. The CSS-based system is too expensive. Thus, this chapter introduced a localization method based on received signal strength index (RSSI). 


\begin{tabular}{|c|c|c|c|c|c|c|c|c|}
\hline & \multicolumn{2}{|c|}{ True position } & \multicolumn{3}{|c|}{ cc2431 } & \multicolumn{3}{|c|}{ New method } \\
\hline & $\mathrm{x}$ & $\mathrm{y}$ & $\mathrm{x}$ & $\mathrm{y}$ & MSE & $x$ & $\mathrm{y}$ & MSE \\
\hline \multirow{10}{*}{ Test1 } & 7.80 & 5.40 & 5.50 & 9.50 & 4.70 & $5 . .93$ & 3.82 & 2.44 \\
\hline & 7.80 & 5.40 & 5.50 & 9.75 & 4.92 & 5.87 & 3.80 & 2.51 \\
\hline & 7.80 & 5.40 & 5.50 & 9.75 & 4.92 & 5.87 & 3.80 & 2.51 \\
\hline & 7.80 & 5.40 & 5.50 & 9.75 & 4.92 & 5.87 & 3.80 & 2.51 \\
\hline & 7.80 & 5.40 & 5.50 & 9.50 & 4.70 & 5.83 & 3.80 & 2.54 \\
\hline & 7.80 & 5.40 & 5.50 & 9.50 & 4.70 & 5.83 & 3.87 & 2.49 \\
\hline & 7.80 & 5.40 & 5.50 & 9.50 & 4.70 & 5.83 & 3.80 & 2.54 \\
\hline & 7.80 & 5.40 & 5.50 & 9.50 & 4.70 & 5.90 & 3.79 & 2.49 \\
\hline & 7.80 & 5.40 & 5.50 & 9.50 & 4.70 & 5.83 & 3.87 & 2.49 \\
\hline & 7.80 & 5.40 & 5.50 & 9.50 & 4.70 & 5.90 & 3.84 & 2.46 \\
\hline \multirow{10}{*}{ Test2 } & 5.40 & 5.40 & 9.50 & 8.00 & 4.85 & 6.28 & 3.25 & 2.32 \\
\hline & 5.40 & 5.40 & 9.50 & 8.50 & 5.14 & 6.41 & 3.11 & 2.50 \\
\hline & 5.40 & 5.40 & 9.50 & 8.50 & 5.14 & 6.41 & 3.11 & 2.50 \\
\hline & 5.40 & 5.40 & 9.50 & 8.50 & 5.14 & 6.41 & 3.03 & 2.58 \\
\hline & 5.40 & 5.40 & 9.50 & 8.50 & 5.14 & 6.41 & 3.03 & 2.58 \\
\hline & 5.40 & 5.40 & 9.50 & 8.50 & 5.14 & 6.41 & 3.03 & 2.58 \\
\hline & 5.40 & 5.40 & 9.50 & 8.50 & 5.14 & 6.41 & 3.03 & 2.58 \\
\hline & 5.40 & 5.40 & 9.50 & 8.50 & 5.14 & 6.41 & 3.03 & 2.58 \\
\hline & 5.40 & 5.40 & 9.50 & 8.50 & 5.14 & 6.27 & 3.15 & 2.42 \\
\hline & 5.40 & 5.40 & 9.50 & 8.50 & 5.14 & 6.27 & 3.15 & 2.42 \\
\hline \multirow{10}{*}{ Test3 } & 5.40 & 5.40 & 13.50 & 14.75 & 11.47 & 8.25 & 3.29 & 1.17 \\
\hline & 9.00 & 4.20 & 13.50 & 14.75 & 11.47 & 8.25 & 3.29 & 1.17 \\
\hline & 9.00 & 4.20 & 13.50 & 14.50 & 11.24 & 8.56 & 3.25 & 1.05 \\
\hline & 9.00 & 4.20 & 13.50 & 14.50 & 11.24 & 8.56 & 3.25 & 1.05 \\
\hline & 9.00 & 4.20 & 13.50 & 14.50 & 11.24 & 8.25 & 3.29 & 1.17 \\
\hline & 9.00 & 4.20 & 13.25 & 14.75 & 11.37 & 8.25 & 3.29 & 1.17 \\
\hline & 9.00 & 4.20 & 13.50 & 14.50 & 11.24 & 8.25 & 3.29 & 1.17 \\
\hline & 9.00 & 4.20 & 13.50 & 14.75 & 11.47 & 8.56 & 2.94 & 1.34 \\
\hline & 9.00 & 4.20 & 13.25 & 14.50 & 11.14 & 8.25 & 3.01 & 1.41 \\
\hline & 9.00 & 4.20 & 13.25 & 14.25 & 10.91 & 8.25 & 3.29 & 1.17 \\
\hline
\end{tabular}

Table 2: Comparison of performance between cc2431 and new method

The algorithms introduced in this chapter update the signal attenuation parameter in real time and calculate the distances between reference nodes and mobile tag. The algorithms have been implemented in ubiquitous ZigBee (2.4 GHz RF communication system) sensor network. The hardware equipment required for the test was developed and tested in office environment. From the comparisons with existing localization chipset Chipcon cc2431, we found that the proposed algorithm (the first method) located the position of an object more accurately than cc2431 as time passed. The second method estimates the position of the tag very fast and accurately. The second method estimates the position much faster than the first method and estimates the position accurately; four cases out of five were better than cc2431 and one case is slightly worse than cc2431. Thus, we conclude from experimental tests that the first method is particularly useful for the position estimation of the stationary 
object, and the second method is practically useful for the fast and reliable position estimation of slowly moving object.

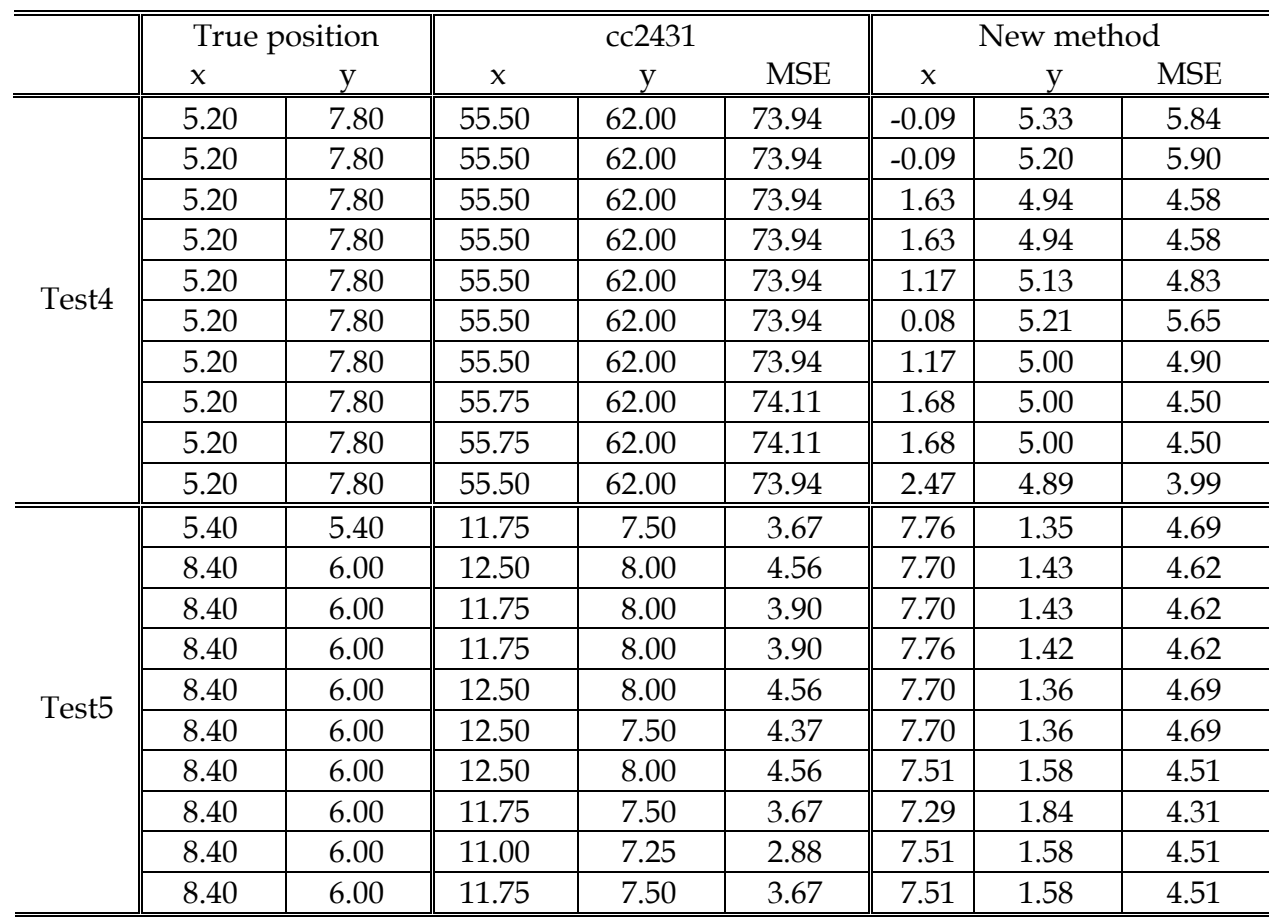

Table 3: Comparison of performance between cc2431 and new method (cont.)

Note that since the methods introduced in this chapter are RSSI-based method, the system is very simple and the implementation cost is much cheaper than TOA and TDOA-based methods, such as Ubisense systems and CSS systems. For a more comprehensive overview and experimental test results of WSN-based localization systems, it is recommended to refer to (Ahn \& Yu, 2006; Ahn et al, 20071; Ahn et al, 20072; Ahn et al, 20081; Ahn \& Yu, 20082; Ahn \& Yu, 20083; Ahn \& Yu, 20084; Hur \& Ahn, 2008).

\section{Acknowledgement}

The work of this chapter was supported in part by the IT R\&D program of Korea MIC (Ministry of Information and Communication) and IITA (Institute for Information Technology Advancement) [2005-S-092-02, USN-based Ubiquitous Robotic Space Technology Development], in part by the financial support from Korea Science and Engineering Foundation [KOSEF, Project No. R01-2008-000-10031-0], and in part by a grant from the institute of Medical System Engineering (iMSE) in the GIST of Korea. 


\section{References}

Ahn, H.-S. \& Yu, W. (2006). Wireless localization network for a ubiquitous robotic space: Background and concept, Proceedings of the 3rd Int. Conf. on Ubiquitous Robots and Ambient Intelligence, pp. 187-192, Seoul, Korea, Nov. 2006

Ahn, H.-S. ; Yu, W. \& Lee, J.-Y. (2007)1'. Wireless localization network for ubiquitous robotic space: Approaches and experimental test, Proceedings of the IEEE International Symposium on Robot and Human Interactive Communication, pp. 1-6, Jeju, Korea, Aug. 2007

Ahn, H.-S.; Lee, J.-Y.; Yu, W. \& Han, K.-S. (2007)22. Indoor localization technique for intelligent robotic space (written in Korean). ETRI's New Technologies, Vol. 22, No., (2007), pp. 48-57

Ahn, H.-S. ; Hur, H. \& Choi, W.-S. (2008)1. One-way ranging without time synchronization for CSS-based indoor localization, Proceedings of the IEEE Int. Conf. on Industrial Informatics, pp. 1-6, Daejeon, Korea, July 2008

Ahn, H.-S. \& Yu, W. (2008)2. Wireless localization network for indoor service robots, Submitted to the IEEE/ASME Int. Conf. Mechatronics and Embedded Systems and Applications, pp. 1 -6, Beijing, China, Oct. 2008

Ahn, H.-S. \& Yu, W. (2008)3. Reference tag-based indoor localization technique, Proceedings of the IFAC World Congress, pp. 1-6, Seoul, Korea, July 2008

Ahn, H.-S. \& Yu, W. (2008) ${ }^{4}$. Environmental-adaptive RSSI-based indoor localization Submitted to IEEE Trans. on Automation Science and Engineering, 2008

Bhargava, V. \& Sichitiu, M. L. (2005). Physical authentication through localization in wireless local area networks, Proceedings of the IEEE Global Telecommunications Conference, pp. 2658-2662, 2005

Bocquet, M.; Loyez, C. \& Benlarbi-Delai, A. (2005). Using enhanced-TDOA measurement for indoor positioning. IEEE Microwave and Wireless Components Letters, Vol. 15, No. 10, (2005), pp. 612-614

Chae, H. ; Lee, Y. ; Yu, W. \& Doh, N. L. (2005). StarLITE: a new artificial landmark for the navigation of mobile robots, Proceedings of the 1st Japan-Korea Joint Symposium on Network Robot Systems, pp. 1-5, Kyoto, Japan

Crepaldi, M. (2005). Analysis, design and simulation of an UWB receiver for indoor localization (Ph.D. thesis), Politecnico Di Torino

Elnahrawy, E.; Li, X. \& Martin, R.-P. (2004). Using area-based presentations and metrics for localization systems in wireless LANs, Proceedings of the 29th Annual IEEE International Conference on Local Computer Networks, pp. 650-657, 2004

Fontana, R.-J., Richley, E., \& Barney, J. (2003). Commercialization of an ultra wideband precision asset location system, Proceedings of the 2003 IEEE Conference on Ultra Wideband Systems and Technologies, pp. 369-373, 2003

Gezici, S.; Tian, Z.; Giannakis, G. B.; Kobayashi, H.; Molisch, A. F., Vincent, H. \& Sahinoglu, Z. (2005). Localization via ultra-wideband radios. IEEE Signal Processing Magazine, Vol. 22, No. 4, pp. 70-84, 2005

Gifford, S. (2005). Experiences with location sensing systems at the University of Michigan, Proceedings of the 2005 NSF CISE/CNS Infrastructure Experience Workshops, pp. 1-5, Urbana, Illinois, 2005 
Guoping, Z. \& Rao, S. V. (2005). Position localization with impulse ultra wide band, Proceedings of the IEEE/ACES International Conference on Wireless Communications and Applied Computational Electromagnetics, pp. 17-22, Honolulu, Hawaii, 2005

Gustafsson, F. \& Gunnarsson, F. (2005). Mobile positioning using wireless networks. IEEE Signal Processing Magazine, Vol. 22, No. 4, (2005), pp. 41-53

Hatami, A. \& Pahlavan, K. (2006). Performance comparison of RSS and TOA indoor geolocation based on UWB measurement of channel characteristics, Proceedings of the IEEE 17th International Symposium on Personal, Indoor and Mobile Radio Communications, pp. 1-6, Helsinki, Finland

He, T.; Huang, C.; Blum, B. M.; Stankovic, J. A.\& Abdelzaher, T. (2003). Range-free localization schemes for large scale sensor networks, Proceedings of the Annual International Conference on Mobile Computing and Networking, pp. 81-95, San Diego, CA, 2003

Hur, H. \& Ahn, H.-S. Hybrid-style wireless localization network for indoor mobile robot applications, Accepted by the US-Korea Conf. on Science, Technology, and Entrepreneurship, pp. 1-5, San Diego, CA, Aug. 2008

Hightower, J. \& Borriello, G. (2001). Location systems for ubiquitous computing. Computer, Vol. 34, No. 8 (2001), pp. 57-66.

Ingram, S. J. ; Harmer, D. \& Quinlan, M. (2004). Ultrawideband indoor positioning systems and their use in emergencies, Proceedings of the 2004 Position Location and Navigation Symposium, pp. 706-715, 2004.

Irahhauten, Z.; Nikookar, H. \& Janssen, G. J. M. (2004). An overview of ultra wide band indoor channel measurements and modeling. IEEE Microwave and Wireless Components Letters, Vol. 14, No. 6, (2004), pp. 386-388

Ladd, A. M.; Bekris, K. E.; Rudys, A. P.; Wallach, D. S. \& Kavraki, L. E. (2004). On the feasibility of using wireless Ethernet for indoor localization. Name IEEE Trans. Robotics and Automation, Vol. 20, No. 3, (2004), pp. 555-559

Larson, L. ; Laney, D. \& Jamp, J. (2003). An overview of hardware requirements for \{UWB\} systems: interference issues and transceiver design implications, Proceedings of the IEEE Military Communications Conference, pp. 863-867, Boston, MA, 2003

Li, B.; Wang, Y.; Lee, H. K., Dempster, A. \& Rizos, C. (2005). Method for yielding a database of location fingerprintings in WLAN. IEE Proceedings-Communications, Vol. 152, No., (2005), pp. 580-586

Lin, Y.-H.; Jan, I-C.; Ko, P. C.; Chen, Y.-Y.; Wong, J.-M. \& Jan, G.-J. (2004). A wireless PDAbased physiological monitoring system for patient transport. IEEE Trans. Inf. Tech. In Biomedicine, Vol. 8, No. 4, (2004), pp. 439-447

Oppermann, I.; Hamalainen, M. \& Iinatti, J. (2004). UWB Theory and Applications, John Wiley \& Sons, West Sussex, England

Oppermann, I.; Stoica, L.; Rabbachin, A.; Shelby, Z. \& Haapola, J. (2004). UWB wireless sensor networks: UWEN-A practical examples. IEEE Communications Magazine, Vol. 42, No., (2004), pp. S27-S32.

Patwari, N.; Ash, J. N.; Kyperountas, S.; Hero, A. O.; Moses, R. L.; \& Correal, N. S. (2005). Locating the nodes. IEEE Signal Processing Magazine, Vol. 22, No. 4, (2005), pp. 54-69

Peyrard, F.; Soutou, C. \& Mercier, J.-J. (2000). Mobile stations localization in a WLAN, Proceedings of the 25th Annual IEEE Conference on Local Computer Networks, pp. 136142, Tampa, FL, 2000. 
Ping, S. (2003). Delay measurement time synchronization for wireless sensor networks, Technical Report, IRB-TR-03-013, Intel Research Berkeley Lab, June 2003

Reed, J. H. (2005). An Introduction to Ultra Wideband Communication Systems, Prentice Hall, Upper Saddle River, NJ

Rehim, M. A. A. A. Y. A. (2004). HORUS: A WLAN-based indoor location determination system (Ph.D. thesis), University of Maryland

Ruiz, B. Q.; Vazquez, A. A.; Rubio, M. L. \& Gareia, J. L. (2005). Impulse radio $\{U W B\}$ system architecture for smart wireless sensor networks, Proceedings of the 2nd International Workshop on Networking with Ultra Wide Band and Ultra Wide Band for Sensor Networks, pp. 35-39, Rome, Italy, 2005

Sayed, A. H.; Tarighat, A. \& Khajehnouri, N. (2005). Networked-based wireless location. IEEE Signal Processing Magazine, Vol. 22, No. 4, (2005), pp. 24-40

Ssu, K.-F.; Ou, C.-H., \& Jiau, H.-C. (2005). Localization with mobile anchor points in wireless sensor networks. IEEE Trans. Vehicular Tech., Vol. 54, No. 3 (2005), pp. 1187-1197

Sun, G.; Chen, J.; \& Guo, W. (2005). Signal processing techniques in network-aided positioning. IEEE Signal Processing Magazine, Vol. 22, No. 4, (2005), pp. 12-23

Xiang, Z.; Song, S.; Chen, J.; Wang, H.; Huang, J. \& Gao, X. (2004). A wireless LAN-based indoor positioning technology. IBM J. Res. E Dev, Vol. 48, No. 5, (2004)

Yu, K., Montillet, J.-P., \& Rabbachin, A. (2006). UWB location and tracking for wireless embedded networks. Signal Processing, Vol. 86, No., (2006), pp. 2153-2171

Yu, W. ; Chae, H. ; Lee, J.-Y. ; Doh, N. L. \& Cho, Y.-J. (2006). Robot localization network for development of ubiquitous robotic space, Proceedings of the 2006 International Symposium on Flexible Automation, Osaka Japan, 2006

Yu, W.; Lee, J.-Y.; Ahn, H.-S.; Ha, Y.-G.; Jang, M.; Sohn, J.-C. \& Kwon, Y.-M (2008). Design and implementation of a ubiquitous robotic space using wireless sensor network and IT infrastructure. Submitted to IEEE Trans. on Automation Science and Engineering

Zhang, M.; Zhang, S.; Cao, J. \& Mei, H. (2006). A novel indoor localization method based on received signal strength using discrete Fourier transform, Proceedings of the First International Conference on Communications and Networking in China, pp. 1-5, Beijing, China 


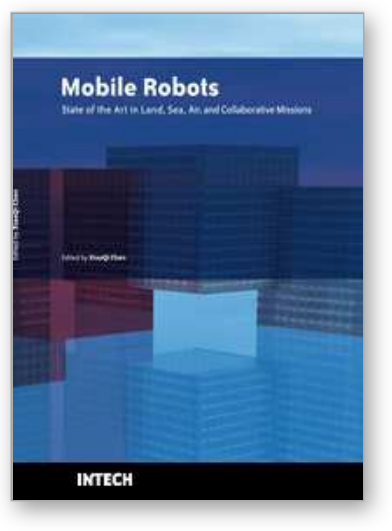

\author{
Mobile Robots - State of the Art in Land, Sea, Air, and \\ Collaborative Missions
}

Edited by XiaoQiChen

ISBN 978-953-307-001-8

Hard cover, 335 pages

Publisher InTech

Published online 01, May, 2009

Published in print edition May, 2009

Since the introduction of the first industrial robot Unimate in a General Motors automobile factory in New Jersey in 1961, robots have gained stronger and stronger foothold in the industry. In the meantime, robotics research has been expanding from fix based robots to mobile robots at a stunning pace. There have been significant milestones that are worth noting in recent decades. Examples are the octopus-like Tentacle Arm developed by Marvin Minsky in 1968, the Stanford Cart crossing a chair-filled room without human assistance in 1979, and most recently, humanoid robots developed by Honda. Despite rapid technological developments and extensive research efforts in mobility, perception, navigation and control, mobile robots still fare badly in comparison with human abilities. For example, in physical interactions with subjects and objects in an operational environment, a human being can easily relies on his/her intuitively force-based servoing to accomplish contact tasks, handling and processing materials and interacting with people safely and precisely. The intuitiveness, learning ability and contextual knowledge, which are natural part of human instincts, are hard to come by for robots. The above observations simply highlight the monumental works and challenges ahead when researchers aspire to turn mobile robots to greater benefits to humankinds. This book is by no means to address all the issues associated mobile robots, but reports current states of some challenging research projects in mobile robotics ranging from land, humanoid, underwater, aerial robots, to rehabilitation.

\title{
How to reference
}

In order to correctly reference this scholarly work, feel free to copy and paste the following:

Hyo-Sung Ahn and Wonpi Yu (2009). Indoor Localization Techniques Based on Wireless Sensor Networks, Mobile Robots - State of the Art in Land, Sea, Air, and Collaborative Missions, XiaoQiChen (Ed.), ISBN: 978953-307-001-8, InTech, Available from: http://www.intechopen.com/books/mobile-robots-state-of-the-art-inland-sea-air-and-collaborative-missions/indoor-localization-techniques-based-on-wireless-sensor-networks

\section{INTECH}

open science | open minds

\section{InTech Europe}

University Campus STeP Ri

Slavka Krautzeka 83/A

51000 Rijeka, Croatia

Phone: +385 (51) 770447

Fax: +385 (51) 686166

\section{InTech China}

Unit 405, Office Block, Hotel Equatorial Shanghai

No.65, Yan An Road (West), Shanghai, 200040, China 中国上海市延安西路65号上海国际贵都大饭店办公楼 405 单元

Phone: +86-21-62489820

Fax: +86-21-62489821 
www.intechopen.com 
(C) 2009 The Author(s). Licensee IntechOpen. This chapter is distributed under the terms of the Creative Commons Attribution-NonCommercialShareAlike-3.0 License, which permits use, distribution and reproduction for non-commercial purposes, provided the original is properly cited and derivative works building on this content are distributed under the same license. 